\title{
The inflammatory response in transgastric surgery: gastric content leak leads to localized inflammatory response and higher adhesive disease
}

\author{
Sonia L. Ramamoorthy · Jeffrey K. Lee $\cdot$ Linda Luo $\cdot$ Yoav Mintz $\cdot$ \\ John Cullen · David W. Easter · Michelle K. Savu · Alana Chock • \\ John Carethers · Santiago Horgan · Mark A. Talamini
}

Received: 6 December 2008/ Accepted: 20 June 2009/Published online: 18 August 2009

(C) The Author(s) 2009. This article is published with open access at Springerlink.com

\begin{abstract}
Background Risk of gastric spillage during transgastric surgery is a potential complication of NOTES procedures. The aim of this study was to determine risk outcomes from gastric spillage in a rat survival model by measuring local and systemic inflammatory markers, adhesive disease, and morbidity.

Methods We performed a minilaparotomy with needle aspiration of $2 \mathrm{ml}$ of gastric contents mixed with $2 \mathrm{ml}$ of sterile saline (study group, SG) or $4 \mathrm{ml}$ of sterile saline (control group, CG) injected into the peritoneal cavity of 60 male rats. Inflammatory markers (TNF $\alpha$, IL-6, and IL10) were analyzed at $1,3,6$, and $24 \mathrm{~h}$ postoperatively by obtaining plasma levels and peritoneal washings. At necropsy, the peritoneal cavity was examined grossly for adhesions.
\end{abstract}

Presented at the Society of American Gastrointestinal and Endoscopic Surgeons, Philadelphia, Pennsylvania, 9-12 April 2008.

Jeffrey K. Lee is a co-first author.

S. L. Ramamoorthy $\cdot$ L. Luo $\cdot$ Y. Mintz $\cdot$ J. Cullen

D. W. Easter $\cdot$ M. K. Savu $\cdot$ A. Chock $\cdot$ S. Horgan .

M. A. Talamini

Center for the Future of Surgery, Department of Surgery,

University of California, San Diego Medical Center,

San Diego, CA, USA

J. K. Lee · J. Carethers

Department of Medicine, University of California,

San Diego Medical Center, San Diego, CA, USA

S. L. Ramamoorthy $(\bowtie)$

UCSD Moores' Cancer Center, 3855 Health Sciences Drive,

Suite 2073, La Jolla, CA 92093-0987, USA

e-mail: sramamoorthy@ucsd.edu
Results Adhesions were seen more frequently in the SG versus the CG $(100 \%$ vs. $33.3 \%, p<0.014)$. There was a significant difference in the peritoneal TNF $\alpha$ levels in the SG compared with the $\mathrm{CG}$, which peaked $1 \mathrm{~h}$ after surgery $(p<0.02)$. Both peritoneal IL-6 and IL-10 levels were higher in the SG versus the $\mathrm{CG}$, which peaked $3 \mathrm{~h}$ after surgery $(p<0.005$ and $p<0.001$, respectively). All peritoneal inflammatory markers returned to undetectable levels at $24 \mathrm{~h}$ for both groups. Plasma cytokines were undetectable at all time intervals.

Conclusion The inflammatory response was found to be a localized and not systemic event, with plasma cytokine levels remaining normal while peritoneal washings revealed a brisk, short-lived localized inflammatory response. There was a significantly higher rate of adhesive disease in the SG compared with the CG; this, however did not translate into a difference in apparent clinical outcome. We conclude that gastric leakage in this NOTES rodent model induces a localized inflammatory response, followed by mild to moderate adhesive disease. This may be important in human NOTES.

Keywords Transgastric surgery $\cdot$ Natural orifice surgery · NOTES

In 1902, Georg Kelling of Dresden performed the first laparoscopic procedure in dogs and in 1910 Jacobaeus of Sweden reported the first laparoscopic operation in humans [1]. Not since then has a new approach to surgical entry into the abdomen been introduced until recently with natural orifice surgery. While the early reports of uneventful transgastric surgery (TGS) in animals and humans have been encouraging, the approach is met with both enthusiasm and trepidation. "No scar" surgery in its broadest 
sense should be a step forward in minimizing the deleterious local and systemic effects of an operation and, in doing so, promote early recovery and return to baseline health. Thus, studying the inflammatory effects of transgastric surgery becomes imperative to determine its value as an alternative to conventional approaches for surgically treatable disease.

A significant amount of research and literature exists that evaluates the physiologic and inflammatory responses to laparoscopic surgery. Specifically, for many disease processes (e.g., colon cancer), minimally invasive techniques have been shown to benefit patients by reducing the immunosuppression associated with major surgery and potentially impacting survival [2]. Other studies have demonstrated that minimally invasive techniques create less of an inflammatory response as evidenced by a reduction in proinflammatory mediators [3-5]. A natural tendency would be to draw direct comparisons between TGS and laparoscopy; however, the major difference is that TGS has the potential for intra-abdominal contamination via the organ that is used to obtain peritoneal access. Early animal studies at our institution revealed that a significant amount of gastric secretions can leak into the peritoneal cavity during TGS depending on the length of operation and closure technique. The aim of this study was to determine the risk of sepsis from gastric spillage in a rat survival model by measuring local and systemic inflammatory markers, adhesive disease, and morbidity.

\section{Materials and methods}

Animals

The study was conducted after approval and supervision from the University of California, San Diego Institute for Animal Care and Use Committee (IACUC Protocol \# S07035). A total of 60 male Sprague-Dawley rats (250$275 \mathrm{~g})$ were obtained from a USDA-approved vendor (Charles River Laboratories, Boston, MA) and underwent 72-h quarantine per IACUC protocol. During this period all animals were caged in standard fashion and given food and water ad libitum and standard husbandry procedures were overseen by UCSD Animal Care. The animals were kept in the institutional animal care facility with room temperature maintained at $25^{\circ} \mathrm{C}$ and an automated $12-\mathrm{h}$ light/dark cycle.

\section{Study protocol}

The animals were randomized and equally divided into a study group $(\mathrm{SG}, N=30)$ and a control group $(\mathrm{CG}$,
$N=30$ ). Using a random-number generator, the clinical research associate randomly and sequentially assigned rats, without blocking or stratification. The SG underwent minilaparotomy and gastric aspiration and spillage into the peritoneal cavity. The CG underwent minilaparotomy with injection of normal saline into the peritoneal cavity. Animals were further divided into five groups of 12 , each of which was sacrificed at different time intervals: 1, 3, 6, and $24 \mathrm{~h}$ and 14 days. Both groups underwent similar preoperative and postoperative care. Animals in the 14-day group were analyzed for adhesion rates.

\section{Preoperative care and anesthesia}

The animals were transferred to a surgical lab facility on the day of the procedure. The animals were weighed and given a study number. Four hours before the procedure animals were fasted, placed in wire-based cages to prevent ingestion of bedding/stool/food, and given access to water ad libitum. Anesthesia was administered via a precision vaporizer. Induction was obtained with $4-5 \%$ inhaled isoflurane and maintained with $2-3 \%$ isoflurane with $100 \%$ oxygen carrying gas. Depth of anesthesia was determined by spontaneous movement, twitching, increased respiratory rate, and increased work at breathing, movement of extremities upon stimulation of plantar surface, and tail and toe pinch. At the minilaparotomy incision site, $0.25 \%$ Marcaine $1 \mathrm{mg} / \mathrm{kg}$ (Astra-Zeneca, Wilmington, DE) was injected for pain control. After anesthesia induction, the left upper quadrant was shaved using electric clippers and sterilely prepped and draped.

\section{Surgery}

After sterile draping of the animals, baseline serum inflammatory markers were obtained using the ventral tail artery or the lateral saphenous vein as access. Local anesthetic was then administered to the incision site in the left upper quadrant of the animal. An incision smaller than $1 \mathrm{~cm}$ was made using sterile instruments and the stomach was exposed. Using a $16 \mathrm{G}$ needle, we aspirated $2 \mathrm{ml}$ of gastric contents into a syringe containing $2 \mathrm{ml}$ of normal saline $(0.9 \%$ solution $)$. This material was then injected into the peritoneal cavity of the study group to mimic gastric spillage during natural orifice surgery. The control group underwent identical minilaparotomy with injection of $4 \mathrm{ml}$ of normal sterile saline $(0.9 \%)$ into the peritoneal cavity. The fascia and muscle were closed using an absorbable suture and the skin was reapproximated using Indermil $^{\circledR}$ Tissue Adhesive (Covidien, Mansfield, MA). Animals were given buprenorphine $0.05 \mathrm{mg} / \mathrm{kg}$ (Reckitt Benckiser Pharmaceuticals, Richmond, VA) subcutaneously prior to recovery for pain control. Total surgery time 
ranged from 10 to $15 \mathrm{~min}$. Animals were recovered in a post-procedure cage, with a heating source and monitoring. Animals were monitored until they were able to maintain sternal recumbency and were normothermic. The animals used for studying cytokine levels at 1,3 , and $6 \mathrm{~h}$ were kept in the surgery center but given food and water ad libitum. The animals used for $24 \mathrm{~h}$ and the longer survival study were returned to the animal facility in standard cages with routine husbandry and food and water ad libitum.

Peritoneal washings and necropsy

At different time intervals (1, 3, 6, and $24 \mathrm{~h})$ animals were sacrificed and serum cytokine levels were drawn using the technique previously mentioned. In addition, peritoneal washing was performed immediately thereafter by instilling $5 \mathrm{cc}$ of sterile normal saline $0.9 \%$ solution into the peritoneal cavity through a small midline incision $(<1 \mathrm{~cm})$. The abdomen was gently massaged and at least $2 \mathrm{ml}$ of fluid was drawn and immediately "cold" centrifuged at $4^{\circ} \mathrm{C}$ at $800 \mathrm{rpm}$ and the supernatant was stored at $-80^{\circ} \mathrm{C}$ for cytokine analysis. This technique had been previously described in humans and animal models for acquisition of local inflammatory data [5]. The animals were then euthanized via heart puncture. Animals that were used for clinical and adhesive disease assessment were kept alive for 14 days. The animals were sacrificed on day 14 using a $\mathrm{CO}_{2}$ chamber. Necropsy was performed through a midline incision and the abdominal cavity assessed for adhesive disease.

\section{Clinical assessment}

Survival animals were followed for 14 days and clinically assessed daily for pain (shivering, hunching over in corner, or labored breathing), daily weight, eating habits, bowel movements, condition of fur, and clinical signs of sepsis. Animals were given pain medication presumptively for the first $24 \mathrm{~h}$ and then as needed as assessed by the study team and the institution's animal care personnel.

\section{Cytokine analysis}

The samples of peritoneal supernatant and plasma were subjected to cytokine analysis for interleukin-6 (IL-6), interleukin-10 (IL-10), and tumor necrosis factor alpha (TNF $\alpha$ ). IL-6, IL-10, and TNF $\alpha$ levels were determined by enzyme-linked immunosorbent assays (ELISA) using commercially available ELISA kits (BD Rat IL-6 ELISA Set Cat. \#550319, BD Rat IL-10 ELISA Set Cat. \#555134, and BD Rat TNF ELISA Set Cat. \#558535, respectively) (BD Biosciences Pharmingen, San Diego, CA).
Necropsy and adhesion analysis

Twelve animals (allocated to our 14-day experiment arm) that were randomized and equally divided into a study group (SG, $N=6)$ and a control group $(\mathrm{CG}, N=6)$ were euthanized by placing the animals in a $\mathrm{CO}_{2}$ chamber until there was complete respiratory arrest and no visible heartbeat. The 12 animals then underwent cardiac puncture to ensure euthanasia and a midline abdominal incision was made and necropsy performed. Adhesive disease was assessed by two independent observers blinded to the group status of the rats. Adhesive disease was considered severe (grade 3) if diffuse adhesions were encountered, moderate (grade 2) if adhesions were present in more than two quadrants in addition to the incision, mild (grade 1) if local adhesions to the incision were seen, and nonexistent (grade $0)$ if no adhesions were seen.

\section{Statistical analysis}

All data were collected in the form of categorical and continuous variables as well as narrative text. Descriptive statistics were used throughout. Statistical analysis was performed using standard means and median equations, and comparative data were analyzed using Student's $t$ test and $\chi^{2}$ test.

\section{Results}

There were $2 / 60$ deaths (3\%) in the study. One rat from the SG died on postoperative day 2. On necropsy, the animal was found to have a diffuse peritonitis throughout the abdomen. The needle aspiration site was sealed, but due to the number of adhesions and exudates around the site it was believed to have leaked and sealed. One rat from the CG died on postoperative day 7. Necropsy did not reveal an intra-abdominal etiology. No significant difference was seen clinically between the two groups with regard to weight gain, activity, or wound-related complications. Of the 12 animals that underwent adhesion analysis, there was a significant difference between the two groups when all adhesions were considered (Table 1). All 6 animals (6/6) in the study group had some level of adhesion compared to $33.3 \%(2 / 6)$ of the animals in the control group $(p<0.014)$. However, if grades 1,2 , and 3 were considered independently, the difference in the number of adhesions found failed to reach significance.

With respect to cytokine profiles after gastric content spillage, $\mathrm{TNF} \alpha$ levels within the peritoneal washings peaked at $1 \mathrm{~h}$ after surgery and gradually returned to undetectable levels by $24 \mathrm{~h}$. The mean peak peritoneal $\mathrm{TNF} \alpha$ levels at $1 \mathrm{~h}$ after surgery in the SG was 
Table 1 Adhesion rates, statistical comparison between control group and study group

\begin{tabular}{lccc}
\hline $\begin{array}{l}\text { Adhesion } \\
\text { grade }\end{array}$ & $\begin{array}{l}\text { Control group } \\
(n=6)\end{array}$ & $\begin{array}{l}\text { Study group } \\
(n=6)\end{array}$ & $\begin{array}{l}p \\
\text { value }\end{array}$ \\
\hline Grade 0 & 4 & 0 & 0.046 \\
Grade 1 & 2 & 4 & 0.248 \\
Grade 2 & 0 & 1 & 0.296 \\
Grade 3 & 0 & 1 & 0.296 \\
Grade 1-3 & 2 & 6 & 0.014 \\
Total & $2 / 6(33 \%)$ & $6 / 6(100 \%)$ & \\
adhesions & & & \\
\hline
\end{tabular}

$184.4 \pm 78 \mathrm{pg} / \mathrm{ml}$ vs. $39.6 \pm 25 \mathrm{pg} / \mathrm{ml}$ in the $\mathrm{CG}(p<$ 0.02) (Fig. 1). Peritoneal IL-6 levels peaked at $3 \mathrm{~h}$ after surgery and returned to undetectable levels by $24 \mathrm{~h}$. The mean peritoneal IL-6 levels at $3 \mathrm{~h}$ after surgery in the SG was $18,985.7 \pm 4933.8 \mathrm{pg} / \mathrm{ml}$ vs. $130 \pm 25 \mathrm{pg} / \mathrm{ml}$ in the CG $(p<0.005)$ (Fig. 2). Peritoneal IL-10 levels also peaked at $3 \mathrm{~h}$ after surgery and returned to undetectable levels by $24 \mathrm{~h}$. The mean peak peritoneal IL-10 levels at $3 \mathrm{~h}$ after surgery in the $\mathrm{SG}$ was $924.2 \pm 332.5 \mathrm{pg} / \mathrm{ml} \mathrm{vs}$.

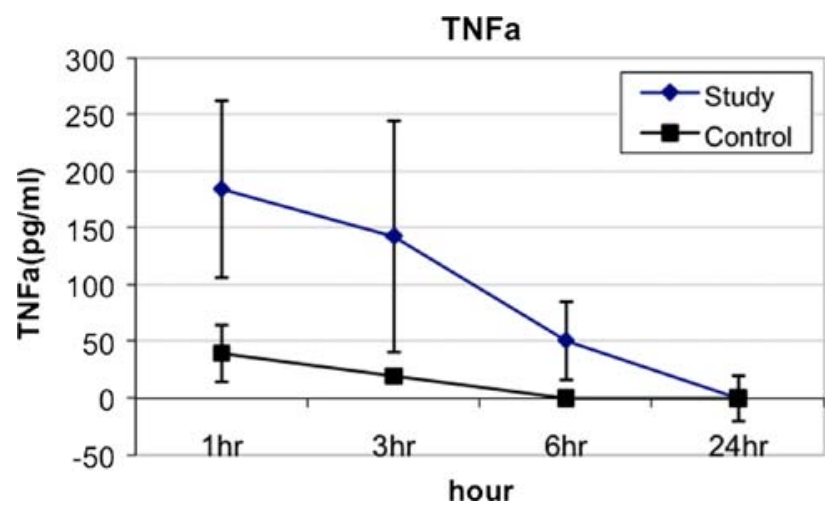

Fig. $1 \mathrm{TNF} \alpha$ levels from peritoneal washings at various time intervals after gastric leakage

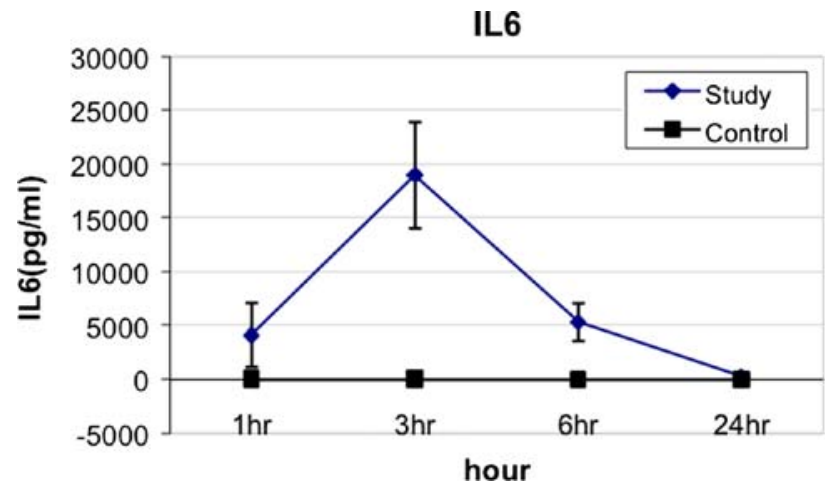

Fig. 2 IL-6 levels from peritoneal washings at various time intervals after gastric leakage

\section{IL10}

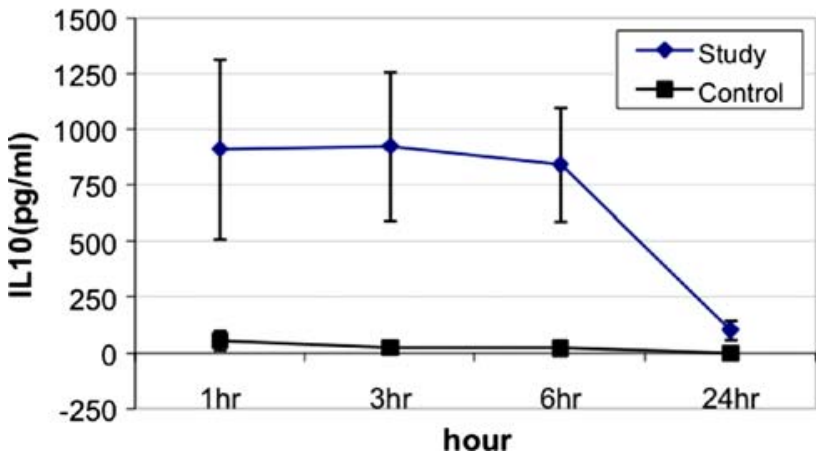

Fig. 3 IL-10 levels from peritoneal washings at various time intervals after gastric leakage

$25 \pm 5 \mathrm{pg} / \mathrm{ml}$ in the CG $(p<0.001)$ (Fig. 3). All plasma cytokines were undetectable at all time intervals.

\section{Discussion}

The measurement of inflammatory mediators during surgical procedures is thought to provide insight into the physiological impact of surgery on the patient; however, little data, outside of oncological data, have been able to show a correlation with clinical outcomes. For cancer patients, the hypothesized benefit of minimally invasive surgery is the reduction in the degree of immunosuppression compared with that of standard open techniques [6, 7]. Differences in immunosuppression translated into differences in cytokine levels have been correlated with prolonged survival for advanced-stage colorectal cancer [6]. In this rat model, we attempted to describe the inflammatory response with clinical outcomes after gastric leakage similar to what we would observe with transgastric surgery. Our rat model demonstrated that there are few systemic manifestations as evidenced by undetectable levels of cytokines following the transgastric leakage and no detectable adverse clinical outcomes from the leakage. The formation of adhesive disease is a natural response to intraabdominal inflammation and infection, and our study demonstrated that transgastric leakage compared with sterile saline causes more adhesive disease within the peritoneal cavity. These findings taken together imply that while transgastric procedures may have minimal systemic impact, there can be a concomitant undetectable inflammatory response within the peritoneal cavity that may result in increased adhesive disease.

Despite our findings, there were several limitations to our study. First, our model was not truly a transgastric model given the lack of endoscopic tools for rats. Instead, we tried to mimic the transgastric effects of gastric spillage with a minilaparotomy. Other approaches were considered 
including gastric lavage with needle puncture of the stomach; however, technical difficulties made the approach challenging. Second, our transgastric approach using minilaparatomy may have caused an elevation of peritoneal cytokines with the stress from the incision and increased adhesion formation; however, increased adhesion formation was found mostly in the study group. Third, our model may not adequately describe the effects of gastric spillage because we mixed $2 \mathrm{ml}$ of gastric contents with $2 \mathrm{ml}$ of normal saline, which diluted our gastric aspirate; thus, the response without dilution could be more extensive. Finally, since our animals were sacrificed after the peritoneal washings were obtained, we were unable to correlate degree of inflammatory response with severity of adhesive disease. Interestingly, as our group has progressed to doing transgastric procedures in humans, the resulting leakage with the advent of better endoscopic instrumentation is minimal by comparison to what was initially seen with our porcine animal model [8].

In conclusion, in this rat model of gastric spillage, the inflammatory response appears to be a short-lived, localized event that did not translate into an adverse clinical outcome. There was, however, a higher rate of mild to moderate adhesive disease in our study group versus the control group, indicating a measurable subclinical localized response to intraperitoneal contamination/manipulation.

Acknowledgment This work was supported by an Ethicon/NOSCAR 2007 research grant.

Open Access This article is distributed under the terms of the Creative Commons Attribution Noncommercial License which permits any noncommercial use, distribution, and reproduction in any medium, provided the original author(s) and source are credited.

\section{References}

1. Nezhat C, Winer WK, Cooper JD, Nezhat F (1989) Endoscopic infertility surgery. J Reprod Med 34(2):127-134

2. Capussotti L, Massucco P, Muratore A, Amisano M, Bima C, Zorzi B (2004) Laparoscopy as a prognostic factor in curative resection for node positive colorectal cancer: Results for a singleinstitution nonrandomized prospective trial. Surg Endosc 18(7):1130-2235

3. Hiki N, Shimizu N, Yamaguchi H, Imamura K, Kami K, Kubota K, Kaminishi M (2006) Manipulation of the small intestine as a cause of the increased inflammatory response after open compared with laparoscopic surgery. Br J Surg 93(2):195-204

4. Matsumoto ED, Margulis V, Tunc L, Taylor GD, Duchene D, Johnson DB, Pearle MS, Cadeddu JA (2005) Cytokine response to surgical stress: comparison of pure laparoscopic, hand-assisted laparoscopic and open nephrectomy. J Endourol 19(9):1140-1145

5. Abramov Y, Ezra Y, Elchalal U, Ben-Shachar I, Fasouliotis SJ, Barak V (2004) Markedly elevated levels of inflammatory cytokines in maternal serum and peritoneal washing during arrested labor. Acta Obstet Gynecol Scand 83(4):358-363

6. Leung KL, Lai PB, Ho RL, Meng WC, Yiu RY, Lee JF, Lau WY (2000) Systemic cytokine response after laparoscopic-assisted resection of rectosigmoid carcinoma: A prospective randomized trial. Ann Surg 231(4):506-511

7. Ordemann J, Jacobi CA, Schwenk W, Stösslein R, Müller JM (2001) Cellular and humoral inflammatory response after laparoscopic and conventional colorectal resections. Surg Endosc 15(6):600-608

8. Horgan S, Cullen JP, Talamini MA, Mintz Y, Ferreres A, Jacobsen GR, Sandler B, Bosia J, Savides T, Easter DW, Savu MK, Ramamoorthy SL, Whitcomb E, Agarwal S, Lukacz E, Dominguez FerrainaP (2009) Natural orifice surgery: initial clinical experience. Surg Endosc 23:1512-1518 